\title{
Study of the Improved Projective Approach and the Variable Separation Solutions in a (2+1)-Dimensional Soliton System
}

\author{
Hong-Gang Liu, Song-Hua Ma \\ College of Science, Lishui University, Lishui 323000, China \\ msh6209@aliyun.com
}

\begin{abstract}
Keywords: Improved projective approach, linear variable separation approach, (2+1)-demensional GCBS system, variable separation solutions
\end{abstract}

\begin{abstract}
The projective approach is a kind of classic, efficient and well-developed method to solve nonlinear evolution equations, the remarkable characteristic of which is that we can have many different ansatzs and therefore, a large number of solutions. In this paper, with the help of the improved projective approach and a linear variable separation approach, some new variable separation solutions of the (2+1)-dimensional Generalized Calogero-Bogoyavlenskii-Schiff system (GCBS) is derived.
\end{abstract}

\section{Introduction}

Modern soliton theory is widely applied in many natural sciences such as chemistry, biology, mathematics, communication, and in particular in almost all branches of physics like fluid dynamics, plasma physics, field theory, optics, and condensed matter physics [1]. The exact solutions of nonlinear partial differential equations(NPDE) are interesting and popular topic in nonlinear physicists and mathematicians, and various methods for obtaining exact solutions of nonlinear system have been proposed, for example, the bilinear method, the standard Painlevé truncated expansion, the method of "coalescence of eigenvalue" or "wavenumbers", the homogenous balance method, and the mapping method [2-5] etc. In the past, Mei and Zhang have obtained exact traveling wave solutions for a nonlinear evolution equation with the Riccari equation ( $\xi^{\prime}=a_{0}+a_{1} \xi+a_{2} \xi^{2}$ )projective method [6].In this paper, by using the Riccari equation projective method, we construct non-traveling wave solutions with $q=l x+m y+n t+R(x, y, t)$ in the (2+1)dimensional Generalized Calogero-Bogoyavlenskii-Schiff (GCBS) system [7] .

$\alpha V_{x t}+2 \beta V_{x} V_{x y}+\beta V_{y} V_{x x}+V_{x x y y}=0$.

where, $\alpha$ and $\beta$ are two constants.

As is well known, to search for the solitary wave solutions for a nonlinear physical model, we can apply different approaches. One of the most efficient methods of finding soliton excitations of a physical model is the so-called mapping approach with variable coefficients. The basic ideal of the algorithm is as follows. For a given nonlinear partial differential equation (NPDE) with the independent variables $x=\left(x_{0}=t, x_{1}, x_{2}, \ldots x_{m}\right)$, and the dependent variable $u$, in the form

$$
P\left(u, u_{t}, u_{x_{i}}, u_{x_{i} y_{j}}, \ldots\right)=0
$$

where $P$ is in general a polynomial function of its arguments, and the subscripts denote the partial derivatives, the solution can be assumed to be in the form,

$$
u=A(x)+\sum_{i=1}^{n}\left\{B_{i}(x) \xi^{i}[q(x)]\right.
$$

with

$$
\xi^{\prime}=a_{0}+a_{1} \xi+a_{2} \xi^{2}
$$


and

$$
q=l x+m y+n t+R(x, y, t)
$$

where $a_{0} a_{1}, a_{2}, l, m, n$ are constants, $R \equiv R(x, y, t)$ is arbitrary function of $(x, y, t)$.Substitute (3), (4) and (5) into the given NPDE and collect coefficients of polynomials of $\xi$, then eliminate each coefficient to derive a set of equations of $A, B_{i}$ and $q$, and solve these equations to obtain $A, B_{i}$ and q. Finally, as (4) is known to possess the solutions[16], one obtains the complex solutions tothe given NPDE.

\section{New Exact Solutions of the GCBS System}

Now we apply the Riccati equation projective approach to (1). By the balancing procedure, ansatz (3) becomes

$$
V=A(x, y, t)+B(x, y, t) \phi(q(x, y, t))
$$

where $A, B$, and $q$ are functions of $(x, y, t)$ to be determined.Substituting(6) and (4) into (1) and collecting coefficients of polynomials of $\phi$, then setting each coefficient to zero, we have

$$
\begin{aligned}
& A=\frac{1}{2 \beta} \int \frac{-\alpha q_{x}^{2} q_{t}+4 a_{0} a_{2} q_{x}^{4} q_{y}-4 a_{1} q_{x} q_{y} q_{x x}-a_{1}^{2} q_{x}^{4} q_{y}-2 q_{x} q_{y} q_{x x x}+q_{x x} q_{y}}{q_{x}^{2} q_{y}} d x, \\
& B=-\frac{4 a_{2} q_{x}}{\beta},
\end{aligned}
$$

$$
q=l x+m y+n t+R(x, y, t), R=\chi(x)+\varphi(y-c d)
$$

Based on the solutions of (4), we can derive the following complex wave solutions of (1):

Case 1 when $a_{0}=1, a_{1}=0, a_{2}=-1$,

$V_{1}=\frac{1}{2 \beta} \int \frac{-\alpha q_{x}^{2} q_{t}+4 a_{0} a_{2} q_{x}^{4} q_{y}-4 a_{1} q_{x} q_{y} q_{x x}-a_{1}^{2} q_{x}^{4} q_{y}-2 q_{x} q_{y} q_{x x x}+q_{x x} q_{y}}{q_{x}^{2} q_{y}} d x$

$-\frac{4 a_{2} q_{x}}{\beta} \tanh (q)$

$V_{2}=\frac{1}{2 \beta} \int \frac{-\alpha q_{x}^{2} q_{t}+4 a_{0} a_{2} q_{x}^{4} q_{y}-4 a_{1} q_{x} q_{y} q_{x x}-a_{1}^{2} q_{x}^{4} q_{y}-2 q_{x} q_{y} q_{x x x}+q_{x x} q_{y}}{q_{x}^{2} q_{y}} d x$

$-\frac{4 a_{2} q_{x}}{\beta} \operatorname{coth}(q)$

Case 2 when $a_{0}=1, a_{1}=0, a_{2}=1$,

$V_{3}=\frac{1}{2 \beta} \int \frac{-\alpha q_{x}^{2} q_{t}+4 a_{0} a_{2} q_{x}^{4} q_{y}-4 a_{1} q_{x} q_{y} q_{x x}-a_{1}^{2} q_{x}^{4} q_{y}-2 q_{x} q_{y} q_{x x x}+q_{x x} q_{y}}{q_{x}^{2} q_{y}} d x$

$-\frac{4 a_{2} q_{x}}{\beta} \tan (q)$,

(11)

Case 3 when $a_{0}=-1, a_{1}=0, a_{2}=-1$,

$$
\begin{aligned}
& V_{4}=\frac{1}{2 \beta} \int \frac{-\alpha q_{x}^{2} q_{t}+4 a_{0} a_{2} q_{x}^{4} q_{y}-4 a_{1} q_{x} q_{y} q_{x x}-a_{1}^{2} q_{x}^{4} q_{y}-2 q_{x} q_{y} q_{x x x}+q_{x x} q_{y}}{q_{x}^{2} q_{y}} d x \\
& -\frac{4 a_{2} q_{x}}{\beta} \cot (q),
\end{aligned}
$$


(12)

Case 4. when $a_{0}=\frac{1}{2}, a_{1}=0, a_{2}=\frac{1}{2}$,

$V_{5}=\frac{1}{2 \beta} \int \frac{-\alpha q_{x}^{2} q_{t}+4 a_{0} a_{2} q_{x}^{4} q_{y}-4 a_{1} q_{x} q_{y} q_{x x}-a_{1}^{2} q_{x}^{4} q_{y}-2 q_{x} q_{y} q_{x x x}+q_{x x} q_{y}}{q_{x}^{2} q_{y}} d x$

$-\frac{4 a_{2} q_{x}}{\beta}(\tan (q)+\sec (q))$

$V_{6}=\frac{1}{2 \beta} \int \frac{-\alpha q_{x}^{2} q_{t}+4 a_{0} a_{2} q_{x}^{4} q_{y}-4 a_{1} q_{x} q_{y} q_{x x}-a_{1}^{2} q_{x}^{4} q_{y}-2 q_{x} q_{y} q_{x x x}+q_{x x} q_{y}}{q_{x}^{2} q_{y}} d x$

$-\frac{4 a_{2} q_{x}}{\beta}(\tan (q)-\sec (q))$

$V_{7}=\frac{1}{2 \beta} \int \frac{-\alpha q_{x}^{2} q_{t}+4 a_{0} a_{2} q_{x}^{4} q_{y}-4 a_{1} q_{x} q_{y} q_{x x}-a_{1}^{2} q_{x}^{4} q_{y}-2 q_{x} q_{y} q_{x x x}+q_{x x} q_{y}}{q_{x}^{2} q_{y}} d x$

$-\frac{4 a_{2} q_{x}}{\beta}(\csc (q)-\cot (q))$,

Case 5 when $a_{0}=-\frac{1}{2}, a_{1}=0, a_{2}=-\frac{1}{2}$,

$V_{8}=\frac{1}{2 \beta} \int \frac{-\alpha q_{x}^{2} q_{t}+4 a_{0} a_{2} q_{x}^{4} q_{y}-4 a_{1} q_{x} q_{y} q_{x x}-a_{1}^{2} q_{x}^{4} q_{y}-2 q_{x} q_{y} q_{x x x}+q_{x x} q_{y}}{q_{x}^{2} q_{y}} d x$

$-\frac{4 a_{2} q_{x}}{\beta}(-\tan (q)+\sec (q))$

$$
\begin{aligned}
& V_{9}=\frac{1}{2 \beta} \int \frac{-\alpha q_{x}^{2} q_{t}+4 a_{0} a_{2} q_{x}^{4} q_{y}-4 a_{1} q_{x} q_{y} q_{x x}-a_{1}^{2} q_{x}^{4} q_{y}-2 q_{x} q_{y} q_{x x x}+q_{x x} q_{y}}{q_{x}^{2} q_{y}} d x \\
& -\frac{4 a_{2} q_{x}}{\beta}(\cot n(q)+\csc (q)),
\end{aligned}
$$

$V_{10}=\frac{1}{2 \beta} \int \frac{-\alpha q_{x}^{2} q_{t}+4 a_{0} a_{2} q_{x}^{4} q_{y}-4 a_{1} q_{x} q_{y} q_{x x}-a_{1}^{2} q_{x}^{4} q_{y}-2 q_{x} q_{y} q_{x x x}+q_{x x} q_{y}}{q_{x}^{2} q_{y}} d x$

$-\frac{4 a_{2} q_{x}}{\beta}(\cot n(q)-\csc (q))$

$V_{11}=\frac{1}{2 \beta} \int \frac{-\alpha q_{x}^{2} q_{t}+4 a_{0} a_{2} q_{x}^{4} q_{y}-4 a_{1} q_{x} q_{y} q_{x x}-a_{1}^{2} q_{x}^{4} q_{y}-2 q_{x} q_{y} q_{x x x}+q_{x x} q_{y}}{q_{x}^{2} q_{y}} d x$

$-\frac{4 a_{2} q_{x}}{\beta} \frac{\cot (q)}{1+\csc (q)}$,

(19)

$$
\begin{aligned}
& V_{12}=\frac{1}{2 \beta} \int \frac{-\alpha q_{x}^{2} q_{t}+4 a_{0} a_{2} q_{x}^{4} q_{y}-4 a_{1} q_{x} q_{y} q_{x x}-a_{1}^{2} q_{x}^{4} q_{y}-2 q_{x} q_{y} q_{x x x}+q_{x x} q_{y}}{q_{x}^{2} q_{y}} d x \\
& -\frac{4 a_{2} q_{x}}{\beta} \frac{\cot (q)}{1-\csc (q)}
\end{aligned}
$$

(20)

Case 6.w hen $a_{0}=\frac{1}{2}, a_{1}=0, a_{2}=-\frac{1}{2}$ 


$$
V_{13}=\frac{1}{2 \beta} \int \frac{-\alpha q_{x}^{2} q_{t}+4 a_{0} a_{2} q_{x}^{4} q_{y}-4 a_{1} q_{x} q_{y} q_{x x}-a_{1}^{2} q_{x}^{4} q_{y}-2 q_{x} q_{y} q_{x x x}+q_{x x} q_{y}}{q_{x}^{2} q_{y}} d x-\frac{4 a_{2} q_{x}}{\beta}
$$

$(\operatorname{coth}(q)+\operatorname{csch}(q))$,

$$
\begin{aligned}
& V_{14}=\frac{1}{2 \beta} \int \frac{-\alpha q_{x}^{2} q_{t}+4 a_{0} a_{2} q_{x}^{4} q_{y}-4 a_{1} q_{x} q_{y} q_{x x}-a_{1}^{2} q_{x}^{4} q_{y}-2 q_{x} q_{y} q_{x x x}+q_{x x} q_{y}}{q_{x}^{2} q_{y}} d x \\
& -\frac{4 a_{2} q_{x}}{\beta}(\operatorname{coth}(q)-\operatorname{csch}(q)),
\end{aligned}
$$

$$
V_{15}=\frac{1}{2 \beta} \int \frac{-\alpha q_{x}^{2} q_{t}+4 a_{0} a_{2} q_{x}^{4} q_{y}-4 a_{1} q_{x} q_{y} q_{x x}-a_{1}^{2} q_{x}^{4} q_{y}-2 q_{x} q_{y} q_{x x x}+q_{x x} q_{y}}{q_{x}^{2} q_{y}} d x
$$

$-\frac{4 a_{2} q_{x}}{\beta}(\tanh (q)+I \operatorname{sech}(q))$,

(23)

$$
\begin{aligned}
& V_{16}=\frac{1}{2 \beta} \int \frac{-\alpha q_{x}^{2} q_{t}+4 a_{0} a_{2} q_{x}^{4} q_{y}-4 a_{1} q_{x} q_{y} q_{x x}-a_{1}^{2} q_{x}^{4} q_{y}-2 q_{x} q_{y} q_{x x x}+q_{x x} q_{y}}{q_{x}^{2} q_{y}} d x \\
& -\frac{4 a_{2} q_{x}}{\beta}(\tanh (q)-I \sec h(q)),
\end{aligned}
$$

$$
\begin{aligned}
& V_{17}=\frac{1}{2 \beta} \int \frac{-\alpha q_{x}^{2} q_{t}+4 a_{0} a_{2} q_{x}^{4} q_{y}-4 a_{1} q_{x} q_{y} q_{x x}-a_{1}^{2} q_{x}^{4} q_{y}-2 q_{x} q_{y} q_{x x x}+q_{x x} q_{y}}{q_{x}^{2} q_{y}} d x \\
& -\frac{4 a_{2} q_{x}}{\beta} \frac{\tanh (q)}{1+\sec h(q)},
\end{aligned}
$$

$V_{18}=\frac{1}{2 \beta} \int \frac{-\alpha q_{x}^{2} q_{t}+4 a_{0} a_{2} q_{x}^{4} q_{y}-4 a_{1} q_{x} q_{y} q_{x x}-a_{1}^{2} q_{x}^{4} q_{y}-2 q_{x} q_{y} q_{x x x}+q_{x x} q_{y}}{q_{x}^{2} q_{y}} d x$

$-\frac{4 a_{2} q_{x}}{\beta} \frac{\tanh (q)}{1-\sec h(q)}$,

(26)

$V_{19}=\frac{1}{2 \beta} \int \frac{-\alpha q_{x}^{2} q_{t}+4 a_{0} a_{2} q_{x}^{4} q_{y}-4 a_{1} q_{x} q_{y} q_{x x}-a_{1}^{2} q_{x}^{4} q_{y}-2 q_{x} q_{y} q_{x x x}+q_{x x} q_{y}}{q_{x}^{2} q_{y}} d x$

$-\frac{4 a_{2} q_{x}}{\beta} \frac{\tanh (q)}{1+\operatorname{csch}(q)}$,

(27)

$V_{20}=\frac{1}{2 \beta} \int \frac{-\alpha q_{x}^{2} q_{t}+4 a_{0} a_{2} q_{x}^{4} q_{y}-4 a_{1} q_{x} q_{y} q_{x x}-a_{1}^{2} q_{x}^{4} q_{y}-2 q_{x} q_{y} q_{x x x}+q_{x x} q_{y}}{q_{x}^{2} q_{y}} d x$

$-\frac{4 a_{2} q_{x}}{\beta} \frac{\operatorname{coth}(q)}{1-\operatorname{csch}(q)}$

(28)

Case 7. when $a_{0}=1, a_{1}=0, a_{2}=-4$, 
$V_{21}=\frac{1}{2 \beta} \int \frac{-\alpha q_{x}^{2} q_{t}+4 a_{0} a_{2} q_{x}^{4} q_{y}-4 a_{1} q_{x} q_{y} q_{x x}-a_{1}^{2} q_{x}^{4} q_{y}-2 q_{x} q_{y} q_{x x x}+q_{x x} q_{y}}{q_{x}^{2} q_{y}} d x$ $-\frac{4 a_{2} q_{x}}{\beta} \frac{\tanh (q)}{1+\tanh ^{2}(q)}$,

(29)

Case 8. when $a_{0}=1, a_{1}=0, a_{2}=4$,

$$
\begin{aligned}
& V_{22}=\frac{1}{2 \beta} \int \frac{-\alpha q_{x}^{2} q_{t}+4 a_{0} a_{2} q_{x}^{4} q_{y}-4 a_{1} q_{x} q_{y} q_{x x}-a_{1}^{2} q_{x}^{4} q_{y}-2 q_{x} q_{y} q_{x x x}+q_{x x} q_{y}}{q_{x}^{2} q_{y}} d x \\
& -\frac{4 a_{2} q_{x}}{\beta} \frac{\tan (q)}{1-\tan ^{2}(q)},
\end{aligned}
$$

Case 9. when $a_{0}=-1, a_{1}=0, a_{2}=-4$,

$$
\begin{aligned}
& V_{23}=\frac{1}{2 \beta} \int \frac{-\alpha q_{x}^{2} q_{t}+4 a_{0} a_{2} q_{x}^{4} q_{y}-4 a_{1} q_{x} q_{y} q_{x x}-a_{1}^{2} q_{x}^{4} q_{y}-2 q_{x} q_{y} q_{x x x}+q_{x x} q_{y}}{q_{x}^{2} q_{y}} d x \\
& -\frac{4 a_{2} q_{x}}{\beta} \frac{\cot (q)}{1-\cot ^{2}(q)},
\end{aligned}
$$

Case 10. when $a_{0}=1, a_{1}=2, a_{2}=2$,

$$
\begin{aligned}
& V_{24}=\frac{1}{2 \beta} \int \frac{-\alpha q_{x}^{2} q_{t}+4 a_{0} a_{2} q_{x}^{4} q_{y}-4 a_{1} q_{x} q_{y} q_{x x}-a_{1}^{2} q_{x}^{4} q_{y}-2 q_{x} q_{y} q_{x x x}+q_{x x} q_{y}}{q_{x}^{2} q_{y}} d x \\
& -\frac{4 a_{2} q_{x}}{\beta} \frac{\tan (q)}{1-\tan (q)},
\end{aligned}
$$

Case 11. when $a_{0}=1, a_{1}=-2, a_{2}=2$,

$$
\begin{aligned}
& V_{25}=\frac{1}{2 \beta} \int \frac{-\alpha q_{x}^{2} q_{t}+4 a_{0} a_{2} q_{x}^{4} q_{y}-4 a_{1} q_{x} q_{y} q_{x x}-a_{1}^{2} q_{x}^{4} q_{y}-2 q_{x} q_{y} q_{x x x}+q_{x x} q_{y}}{q_{x}^{2} q_{y}} d x \\
& -\frac{4 a_{2} q_{x}}{\beta} \frac{\tan (q)}{1+\tan (q)},
\end{aligned}
$$

Case 12. when $a_{0}=-1, a_{1}=2, a_{2}=-2$

$$
\begin{aligned}
& V_{26}=\frac{1}{2 \beta} \int \frac{-\alpha q_{x}^{2} q_{t}+4 a_{0} a_{2} q_{x}^{4} q_{y}-4 a_{1} q_{x} q_{y} q_{x x}-a_{1}^{2} q_{x}^{4} q_{y}-2 q_{x} q_{y} q_{x x x}+q_{x x} q_{y}}{q_{x}^{2} q_{y}} d x \\
& -\frac{4 a_{2} q_{x}}{\beta} \frac{\cot (q)}{1+\cot (q)},
\end{aligned}
$$

Case 13. when $a_{0}=-1, a_{1=-2}, a_{2}=-2$,

$V_{27}=\frac{1}{2 \beta} \int \frac{-\alpha q_{x}^{2} q_{t}+4 a_{0} a_{2} q_{x}^{4} q_{y}-4 a_{1} q_{x} q_{y} q_{x x}-a_{1}^{2} q_{x}^{4} q_{y}-2 q_{x} q_{y} q_{x x x}+q_{x x} q_{y}}{q_{x}^{2} q_{y}} d x$

$-\frac{4 a_{2} q_{x}}{\beta} \frac{\cot (q)}{1-\cot (q)}$

Case 14. when $a_{0}=0, a_{1}=0, a_{2}=\mathrm{a}$, 
$V_{28}=\frac{1}{2 \beta} \int \frac{-\alpha q_{x}^{2} q_{t}+4 a_{0} a_{2} q_{x}^{4} q_{y}-4 a_{1} q_{x} q_{y} q_{x x}-a_{1}^{2} q_{x}^{4} q_{y}-2 q_{x} q_{y} q_{x x x}+q_{x x} q_{y}}{q_{x}^{2} q_{y}} d x$

$+\frac{4 a_{2} q_{x}}{\beta} \frac{1}{a q+k}$

(36)

with $q=l x+m y+n t+R, R=\chi(x)+\varphi(y-c t)$.Here, $\alpha, \beta, c, a$ and $k$ are constants.

\section{Conclusions}

The projective equation method is a kind of classic, efficient and well-developed method to solve nonlinear evolution equations. In the past, Mei and Zhang have obtained the exact traveling wave solutions for the nonlinear evolution equations such as Gross-Pitavskii equation with the Riccari equation projective approach. In this paper, we extend this approach and construct the variable separation solutions of the (2+1)-dimensional Generalized Calogero-Bogoyavlenskii-Schiff system, which are different from the ones of the previous work. Since the wide applications of the soliton theory, to learn more about the localized excitations and their applications in reality is worthy of study further.

\section{References}

[1] Qian S P, Tian L X (2007) Lie symmetry analysis and reduction of a new integral coupled KdV system. Chin. Phys. B 16(2):303-307

[2] Yang XY, Tang JS (2007) New travelling wave solutions for combined KdV. mKdV equation and (2+1)-dimensional Broer-Kaup-Kupershmidt system. Chin. Phys. B16(2):310-317

[3] Wang YH, Chen Y (2011) Binary bell polynomials bilinear approach to exact periodic wave solutions of (2+1)-dimensional nonlinear evolution equation.Common Theory Phys 56(4):672-678

[4] Ma SH, Wu XH, Fang JP, Zheng CL (2009) New exact solutions for the (3+1)-dimensional Jimbo-Miwa system. Chaos Solutions and Fractals 40(3):1352-1355

[5] Ma SH, Fang JP, Zheng CL (2009) New exact solutions of the (2+1)-dimensional breaking soliton system via an extended mapping method. Chaos Solutions and Fractals 40(1):210-214

[6] Mei JQ, Zhang HQ (2005) New families of soliton and periodic solutions of bose einstein condensation in linear magnetic field and time-dependent laser field. Commune Theory Phys 44(02):209-212 44, 209-212

[7] Zhang HP, Chen Y, Li B (2009) Infinitely many symmetries and symmetry reduction of (2+1)dimensional generalized Calogero-Bogoyavlenskii-Schiff equation. Acta Phys. Sin 58(11):7393-04 